\title{
Cardiac autonomic function and oesophageal acid sensitivity in patients with non-cardiac chest pain
}

G Tougas, R Spaziani, S Hollerbach, V Djuric, C Pang, A R M Upton, E L Fallen, M V Kamath and arterial pressure. ${ }^{3-5}$ These effects are primarily mediated through vagal and sympathetic pathways.

Gastro-oesophageal reflux disease (GORD) is the most common oesophageal aetiology of NCCP. ${ }^{6}$ While most GORD patients have evidence of oesophagitis, up to $40 \%$ with reflux-like symptoms have no evidence of mucosal damage at endoscopy and no evidence of pathological acid reflux during $\mathrm{pH}$ monitoring. ${ }^{7}$ These patients are called "acid sensitive" and may respond to therapies aimed at inhibiting acid secretion. ${ }^{8}$ Many mechanisms have been proposed to explain the symptoms seen in acid sensitive NCCP patients. ${ }^{910}$ These include sensitisation of specific sensory afferent neurones acting as chemoceptive, mechanoceptive, and/or nociceptive receptors, or modulation of the response at the level of the spinal dorsal root. ${ }^{11}$

In recent years there has been increasing evidence to suggest that the autonomic nervous system may modulate visceral sensory perception. ${ }^{12}$ Sympathetically mediated mechanisms are implicated in several chronic pain syndromes. ${ }^{13}$ In addition, there is substantial animal and human data supporting a vagally mediated modulation of visceral nociceptive sensory inputs. ${ }^{14} 15$ The possibility that such mechanisms may be involved in NCCP is yet to be determined. There is no sensitive or specific technique currently available to examine oesophageal autonomic input. Assessing visceral autonomic function can be difficult in humans.

The oesophagus and heart share a very similar innervation. While direct measurements of heart rate and arterial pressure provide some index of autonomic outflow, there are now methods that can provide a more accurate assessment of the balance between sympathetic and vagal activity. One of these approaches examines heart rate variability using power spectral analysis of beat to beat heart rate variability (PSHRV). This technique has been extensively studied and validated to provide an objective assessment of the autonomic outflow to the heart, ${ }^{16}$ including responses to visceral stimuli such as mechanical distention and electrical stimulation. ${ }^{17}$

The aim of our study was to investigate whether there is a difference in autonomic regulatory mechanisms in NCCP patients. In

Abbreviations used in this paper: NCCP, non-cardiac chest pain; PSHRV, power spectral analysis of heart rate variability; HF, high frequency; LF, low frequency; GORD, gastro-oesophageal reflux disease; ECG, electrocardiogram. 
addition, we hypothesised that acid sensitive patients who experience chest pain with acid reflux will have reflex changes in autonomic outflow in association with perception of the noxious stimulus.

\section{Subjects and methods \\ PATIENTS}

We studied 28 consecutive patients (40.5 (10.2) years; 13 women, 15 men) referred to the Gastrointestinal Investigation Unit, McMaster University Medical Centre for oesophageal motility testing as part of their evaluation for recurrent NCCP. All had a prior negative cardiac workup, independently done by cardiologists who were not involved in the present study. Inclusion criteria in the study were based on at least one of the three following investigations: a negative maximal exercise stress test (26 subjects), a negative stress thallium scan (18 patients), or a normal coronary angiogram (11 patients). Pathological reflux was excluded in all patients through a routine 24 hour $\mathrm{pH}$ study $(<4.5 \%$ of study with $\mathrm{pH}<4.0$ ). If acid reflux was associated with either heartburn or NCCP on the 24 hour $\mathrm{pH}$ study, patients were treated with a proton pump inhibitor.

After an overnight fast, patients presented to the gastrointestinal investigation unit. Patients had been required to discontinue acid suppressing medications (omeprazole five days prior to the study, histamine receptor antagonists three days prior to the study, and prokinetic agents 24 hours before test). Antacids were stopped at midnight the evening before. Patients were also asked to abstain from alcohol and tobacco after $10 \mathrm{pm}$ the night before. Medications required for other medical conditions were continued but drugs with a known anticholinergic activity were withheld for 24 hours prior to testing. Patients receiving $\beta$ adrenergic blocking drugs were excluded. Ten healthy volunteers (seven men, three women) without any prior history of either gastro-oesophageal or cardiac complaints were also investigated in a similar fashion. The study was conducted in full agreement with the principles of the declaration of Helsinki (1977) regarding ethical standards for clinical studies in medicine. The McMaster University Medical Centre Research Advisory Group approved the experimental protocol. Written informed consent was obtained from all participants prior to the study.

\section{OESOPHAGEAL MANOMETRY}

A six lumen catheter (diameter $4.7 \mathrm{~mm}$; DentSleeve Pty Ltd, Belair, Australia) was inserted and the $6 \mathrm{~cm}$ Dent sleeve positioned astride the lower oesophageal sphincter. The four proximal side holes were positioned $3,8,13$, and 18 $\mathrm{cm}$ proximal to the lower oesophageal sphincter and the most distal channel, distal to the sleeve, was in the stomach. Each channel was continuously perfused with distilled water $(0.5$ $\mathrm{ml} / \mathrm{min}$ ) using a low compliance pneumohydraulic capillary infusion pump (Mui Scientific, Mississauga, Ontario, Canada). Pressures were amplified, digitised, and recorded using a computerised multichannel system (SmartLab Motility System, Model TDS-SGM, Sandhill Scientific Incorporated, Littleton, Colorado, USA). Contractions in the oesophageal body were measured using 10 swallows of $5 \mathrm{ml}$ of water given at intervals of 30 seconds. Measured indices of oesophageal motility included basal lower oesophageal pressure $(\mathrm{mm} \mathrm{Hg})$, lower oesophageal sphincter relaxation with swallowing $(\%)$, wave amplitude (mm $\mathrm{Hg}$ ), wave velocity $(\mathrm{cm} / \mathrm{s})$, and peak velocity $(\mathrm{cm} / \mathrm{s})$. These indices were measured using commercially available software (SmartGraph; Sandhill Scientific Incorporated, Littleton, Colorado, USA).

\section{ASSESSMENT OF ACID SENSITIVITY}

Following standard oesophageal manometry and wet swallows, oesophageal acid sensitivity was assessed. Using a blinded crossover design, either $0.1 \mathrm{~N} \mathrm{HCl}$ or normal saline was administered into the distal oesophagus $(5 \mathrm{~cm}$ above the lower oesophageal sphincter; rate 10 $\mathrm{ml} /$ minute) for 10 minutes or until chest pain occurred, using a variable pressure volumetric intravenous pump (IVAC, Model 560MEE; IVAC Corporation, San Diego, California, USA). If acid perfusion reproduced the pain for which the patient was being investigated (and not just heartburn), the individual was classified as acid sensitive and asked to rate the pain on a visual analogue scale. Maximum pain was rated as 10 and minimal discomfort as 1 . Subjects were considered acid insensitive if they had no symptoms during acid infusion.

\section{PROTOCOL}

All studies were conducted in a quiet laboratory, the subjects resting supine with a $60^{\circ}$ elevation of the head of the bed. A standard oesophageal manometric study was first performed using the techniques and parameters previously described. The acid perfusion test was then performed. The test was stopped if the subject experienced severe pain during acid infusion but was continued for the full $10 \mathrm{~min}-$ utes if symptoms were felt to be tolerable by the subject. An electrocardiogram (ECG) was recorded continuously throughout the study and for 20 minutes after the end of acid perfusion. PSHRV was subsequently performed using the algorithms and protocols described below.

POWER SPECTRAL ANALYSIS OF HEART RATE VARIABILITY

A continuous ECG was recorded from three leads placed in the standard limb lead II position. Through an ECG amplifier (HewlettPackard, Model 7807C), the signal was then digitised using a 12 bit analogue to digital converter (DATAQ Instruments, Akron, Ohio, USA) and recorded on a Pentium-90 MHz computer (Dell Dimension XPS90; Dell Computer Corporation, Austin, Texas, USA) with a $500 \mathrm{~Hz}$ sampling frequency. The ECG recording obtained could then be digitally played back (Windaq/EX; DATAQ Instruments Inc.) for analysis. Separate recordings were made during the baseline period and during 
Table 1 Comparison of oesophageal manometry data obtained from acid sensitive and insensitive patients

\begin{tabular}{lcc}
\hline Manometric parameter & Acid insensitive & Acid sensitive \\
\hline LOS pressure (mm Hg) & $13.16(3.19)$ & $13.32(1.64)$ \\
\% Relaxation & $85.18(4.88)$ & $84.61(3.05)$ \\
Distal oesophagus (mm Hg) (3 cm above LOS) & $36.67(8.31)$ & $50.42(5.60)$ \\
Oesophageal body 1 (mm Hg) (8 cm above LOS) & $46.67(10.05)$ & $57.00(5.70)$ \\
Oesophageal body 2 (mm Hg) (13 cm above LOS) & $61.44(13.74)$ & $65.89(7.71)$ \\
Proximal oesophagus (mm Hg) (18 cm above LOS) & $46.55(13.66)$ & $55.84(6.99)$ \\
Velocity (cm/s) & $2.62(0.32)$ & $3.20(0.14)$ \\
Peak velocity (cm/s) & $2.24(0.42)$ & $2.27(0.14)$ \\
\hline
\end{tabular}

No significant differences between the two groups for any parameter.

Data are mean (SEM).

LOS, lower oesophageal sphincter.

oesophageal acidification, and for 20 minutes after the end of acid infusion.

Data were analysed using a peak detection algorithm identifying the $\mathrm{R}$ wave of the QRS complexes after all motion artefacts were edited. A QRS detection algorithm was implemented in the software to locate a stable and noise independent fiducial point on the $R$ wave. Following QRS detection, a RR interval series was formed from the continuous ECG data. A beat to beat heart rate variability signal was then computed, and the data re-sampled at $2 \mathrm{~Hz}$ using linear interpolation to obtain an equally sampled time series. A record length of 256 points from the re-sampled signal (128 seconds) was used for power spectral analysis. The mean value of the signal was subtracted and the equally sampled heart rate variability signals were fed through a second order high pass Butterworth filter with a cutoff of $0.02 \mathrm{~Hz}$. A ninth order autoregressive model was then applied to the demeaned filtered heart rate variability data. The computational details of the algorithm for estimating the autoregressive parameters have been previously described. ${ }^{16-19}$ The following indices were computed to characterise the information contained in the power spectrum: maximum power contained in the LF (low frequency $0.02-0.15 \mathrm{~Hz}$ ) and $\mathrm{HF}$ (high frequency $0.15-0.5 \mathrm{~Hz}$ ) bands of the power spectrum. The area subtended by each spectral band was computed by numerically integrating the power therein contained. In addition, the normalised areas under both LF and $\mathrm{HF}$ bands were derived by dividing the integrative power within each band by the total power contained in the entire spectrum. The $\mathrm{LF} / \mathrm{HF}$ ratio was expressed as the ratio of these normalised areas by computing a ratio of the percentage power contained in the low frequency band to that in the high frequency band ( $\mathrm{LF} / \mathrm{HF}$ ratio). Mean heart rate, $\mathrm{LF} / \mathrm{HF}$ power ratio, individual $\mathrm{LF}$ and $\mathrm{HF}$ area power (beats/ $\min )^{2}$, and normalised area were individually measured from all subjects for all experimental conditions.

STATISTICS

All manometric and heart rate variability data were subjected to standard $t$ test and paired $t$ test analysis when appropriate. One way analysis of variance (in order to determine univariate differences between acid sensitive and acid insensitive patients) and repeated measures of analysis of variance (in order to determine any differences between the three phases of the

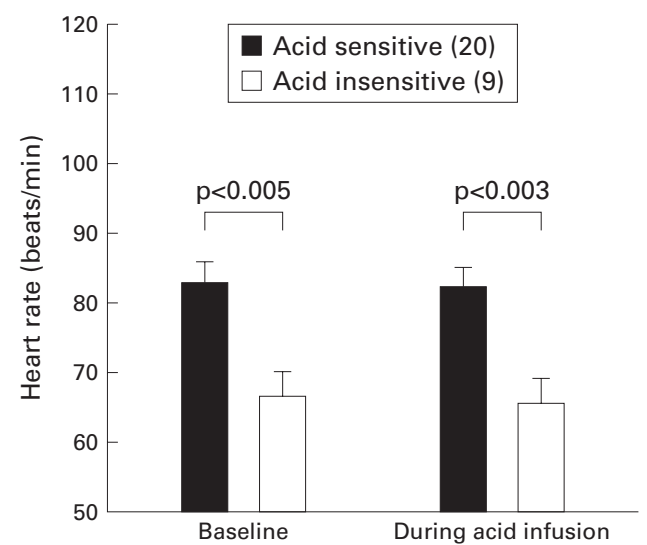

Figure 1 Mean heart rate in acid sensitive and acid insensitive patients with non-cardiac chest pain, recorded before (baseline) and during (acid) oesophageal acid perfusion. Data are mean (SEM).
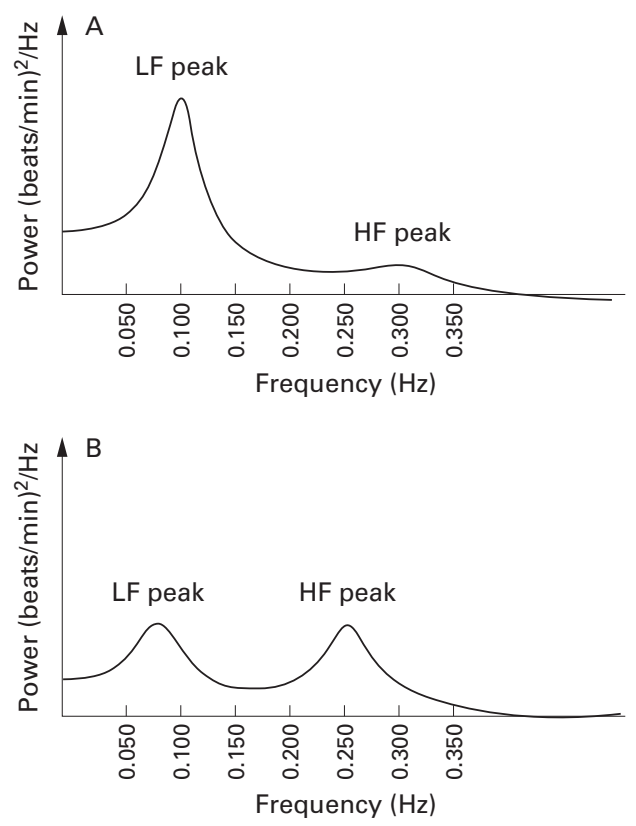

Figure 2 Power spectral analysis of baseline heart rate variability in acid sensitive $(A)$ and insensitive $(B)$ patients with non-cardiac chest pain. A clear preponderance of the low frequency ( $L F$ ) band with only a small high frequency $(H F)$ band is seen in an acid sensitive patient (A) while the acid insensitive patient has a lower LF band and greater $\mathrm{HF}$ band $(B)$.

test) were also used. A stepwise discriminate analysis using power spectral indices and oesophageal motility parameters was used to develop models to predict the outcome of the acid challenge. Linear association between variables was assessed by a Pearson product moment correlation. Statistical significance was set at $p<0.05$. Statistics were performed using the SAS computer software package. All data are expressed as mean (SEM).

\section{Results}

SYMPTOMS AND MANOMETRIC DATA DURING ACID INFUSION

During oesophageal acid infusion, 19/28 $(68 \%)$ patients experienced chest pain $( \pm$ heartburn) and were thus classified as acid sensitive. This group ( $n=19$; eight women, 11 men) tolerated an average of 29.8 (5.2) $\mathrm{ml}$ of 
A

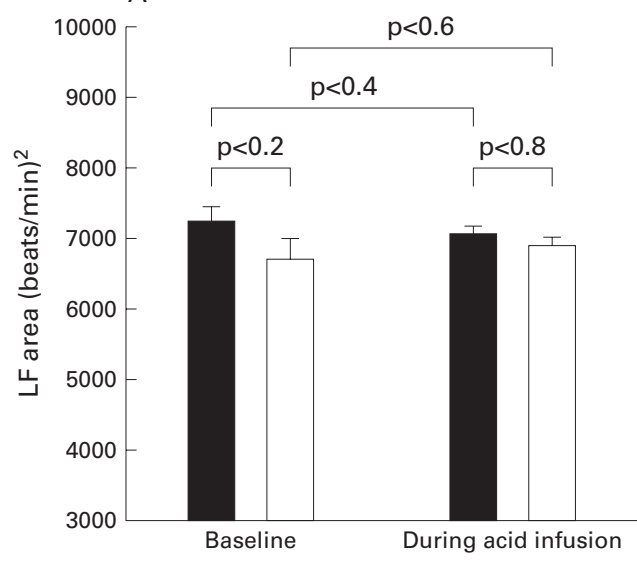

B

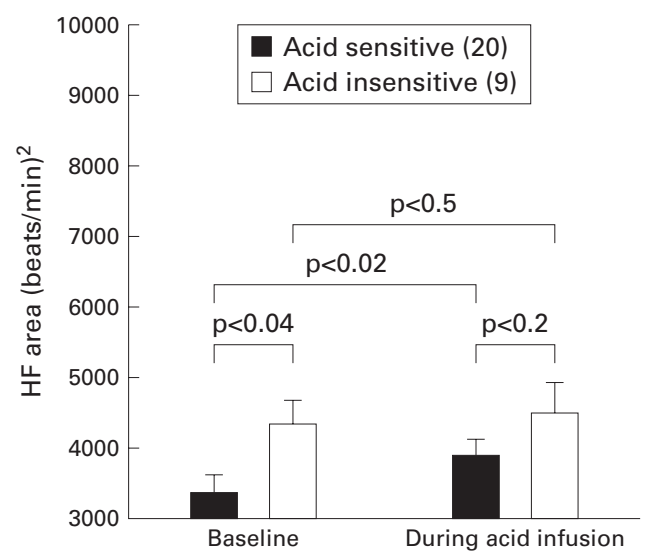

Figure 3 (A) Absolute low frequency (LF) power area at baseline and during oesophageal acid perfusion in acid sensitive and insensitive patients with non-cardiac chest pain. (B) Absolute high frequency (HF) power area at baseline and during oesophageal acid perfusion in acid sensitive and acid insensitive patients with non-cardiac chest pain. Data are mean (SEM).

acid before experiencing symptoms. This compared with $>100 \mathrm{ml}$ of acid without symptoms in acid insensitive individuals and control subjects $(\mathrm{p}<0.05)$. On average, they rated their pain as $6.2(0.3)$ on a scale ranging from 0 to 10 . Nine patients ( $n=9$; four women, five men) did not experience any symptoms during acid infusion (total of $100 \mathrm{ml}$ ) and were thus identified as acid insensitive. None of the control subjects had any symptoms during acid infusion.

Patients comprising the acid sensitive group were significantly older than acid insensitive patients (48 (1.1) v 37.0 (2.4) years; $\mathrm{p}<0.005)$. There was no difference in the oesophageal motility parameters between acid sensitive and acid insensitive patients either at baseline or during oesophageal acid infusion (table 1). There was no association between symptoms and distinct manometric abnormalities in any of the acid sensitive patients.

BASELINE HEART RATE AND HEART RATE

VARIABILITY DATA

There were substantial differences in baseline measures of resting heart rate and heart rate variability. Prior to acid infusion, acid sensitive patients had a higher resting heart rate (82.9 (3.1) beats/ $\mathrm{min}$ ) than acid insensitive patients (66.7 (3.5) beats/min) and healthy controls $(p<0.005)$ (fig 1). Healthy controls had a resting heart rate of $63.1(2.0)$ beats $/ \mathrm{min}(\mathrm{p}<0.005$ $v$ acid sensitive patients, NS $v$ acid insensitive patients). Acid infusion did not alter heart rate in NCCP patients or in healthy controls (fig 1). There was no correlation between age and resting heart rate.

Figure 2 (A, B) shows an example of baseline power spectrum and of the typical shift towards a higher vagal response (HF) during acid infusion in an acid sensitive patient. Prior to acid infusion, there was a trend towards enhanced sympathetic modulation in acid sensitive patients $(7258(210) v 6715 \text { (301) (beats/min) })^{2}$ in acid insensitive individuals) but these values did not reach statistical significance (fig 3A). However, prior to acid infusion, acid sensitive patients had significantly $(\mathrm{p}<0.04)$ lower baseline cardiovagal modulation ( $\mathrm{HF}$ area) of heart rate variability compared with acid insensitive patients (fig 3B). Prior to acid infusion, mean absolute HF power in the acid sensitive group was 3351 (246) v 4317 (336) (beats/min) ${ }^{2}$ in acid insensitive patients $(\mathrm{p}<0.04)$. Expressed in normalised units ( $\%$ of the total AUC), baseline $\mathrm{HF}$ area was significantly less than that of acid insensitive patients (31.1 (1.9)\% $v 38.9$ $(2.3) \%$; $<0.03)$. Consequently, the baseline $\mathrm{LF} / \mathrm{HF}$ power ratio was much greater in acid sensitive patients than in acid insensitive patients $(2.49(0.25) v 1.63(0.13) ; \mathrm{p}<0.01)$ (fig 4).

Healthy controls had comparable baseline values to acid insensitive patients for all parameters examined, including resting heart rate and power spectral variables (fig 5 (A, B)).

We repeated the study in healthy controls, including oesophageal acid infusion and normal saline administration, on two different days using an identical protocol to assess day to day reproducibility. We found that the heart rate and cardioautonomic responses to oesophageal acidification were nearly identical on these two days (fig 5A, B)).

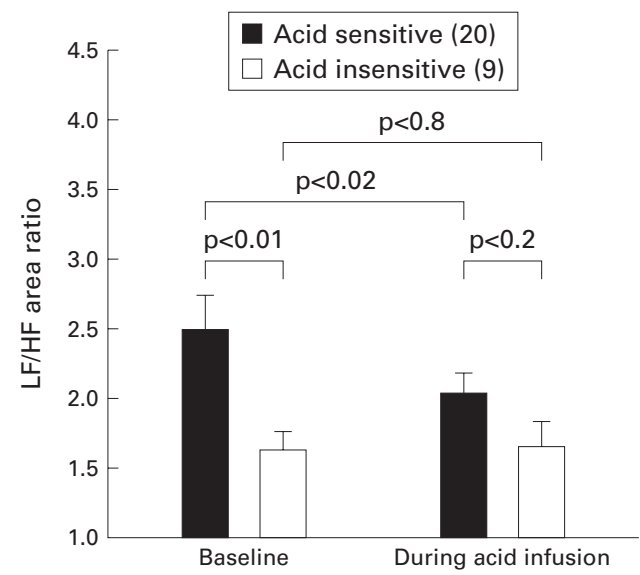

Figure 4 Low frequency/high frequency (LF/HF) area ratio at baseline and during oesophageal acid perfusion in acid sensitive and acid insensitive patients. 

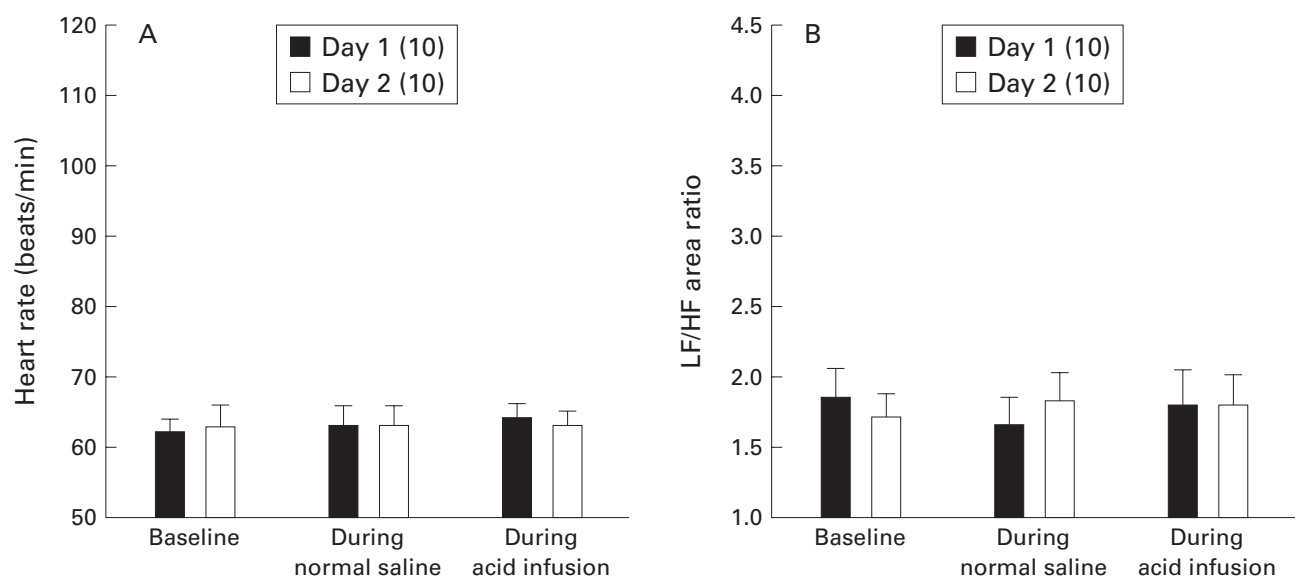

Figure 5 (A) Mean heart rate in matched healthy controls at baseline and during either normal saline or acid perfusion. The protocol was repeated on two days, alternating the order of acid and saline perfusion. Data are mean (SEM). (B) Low frequency/high frequency $(L F / H F)$ area ratio in healthy controls at baseline and during either normal saline or acid perfusion. None of the healthy controls reported symptoms.

HEART RATE AND HEART RATE VARIABILITY DURING ACID INFUSION

During acid perfusion, several distinct changes were noted in acid sensitive but not in acid insensitive patients or in healthy controls. While heart rate did not change significantly throughout the experiment in any of the groups studied (fig 1), there was a significant increase in the vagally modulated $\mathrm{HF}$ area in acid sensitive patients (fig 3B). This increase in the vagally modulated $\mathrm{HF}$ area was not observed either in acid insensitive patients (fig 3B) or in healthy controls (fig 5A). These changes were not due to regression towards the mean in acid sensitive patients as the $\mathrm{HF}$ area returned to its baseline levels in the period immediately following cessation of the acid infusion (3351 (246) (beats $/ \mathrm{min})^{2}$ at baseline, 3853 (258) (beats/min) ${ }^{2}$ during acid infusion, and 3443 (261) (beats $/ \mathrm{min})^{2}$ following the end of acid infusion).

During acid infusion, we did not observe a significant change in the absolute LF area either in acid sensitive or insensitive patients, although there was a trend towards a higher LF area in acid sensitive patients (fig 3A).

In the acid sensitive group, the $\mathrm{LF} / \mathrm{HF}$ area ratio decreased significantly from 2.49 (0.25) to $2.03(0.16)(\mathrm{p}<0.02$, fig 4$)$ during acid infusion but subsequently returned to baseline levels (2.4 (0.1)) within 20 minutes following oesophageal acidification. This was primarily due to the effect of acid infusion on the vagal efferent modulation of heart rate variability as opposed to a change in sympathetic activity.

In healthy controls, neither acid nor normal saline infusion into the oesophagus produced any measurable changes in heart rate or PSHRV parameters (fig 5A, B). None of the healthy controls experienced any symptoms during acid infusion.

\section{Discussion}

In the present study, oesophageal acid infusion increased vagal modulation of heart rate variability in acid sensitive NCCP patients, as evidenced by the significant increase in high frequency power. This cardiac vagal response was only present in patients that experienced symptoms and was not observed in acid insensitive patients or in healthy controls. This is consistent with an ability to mount a vagal inhibitory response independent of alterations in sympathetic activity in acid sensitive NCCP patients. Oesophageal acid infusion produced chest pain in $2 / 3$ patients, supporting the notion that angina-like chest pain can be the result of enhanced oesophageal perception of acid. ${ }^{20}$ Acid perfusion had no effect on heart rate and did not cause dysrhythmia. To the best of our knowledge, this is the first evidence of a vagally mediated cardiac reflex response during oesophageal acidification in patients with NCCP.

We have previously shown that electrical or mechanical oesophageal stimulation also increases reflex vagal efferent activity. ${ }^{17} 2122$ The same stimuli also elicit reproducible cerebral evoked responses. ${ }^{23}$ We have reported similar cerebral evoked responses and increased vagal efferent activity with direct electrostimulation of the left cervical vagus. ${ }^{2324}$

Of interest, NCCP patients who were acid sensitive demonstrated suppressed vagal tone under resting baseline conditions. This impaired balance appears to be associated with a heightened awareness of viscerosensory perception in the absence of any demonstrable abnormality of oesophageal motility. However, this does not per se exclude the possibility that acid sensitive patients may have heightened sympathetic activity as well, insofar as the power contained within the LF band under supine conditions includes both vagal and sympathetic input. ${ }^{16}$

Although the acid sensitive group was younger than the acid insensitive group, the altered autonomic balance during acid infusion was unlikely to be a direct effect of this difference. We have independent evidence that in healthy volunteers within the age range of our study population, there are no substantial differences in power spectral indices. ${ }^{25} 26$

Abnormal vagal function has previously been reported in some patients with pathological acid reflux ${ }^{27}$ but the changes reported 
were primarily correlated with altered motility and not with symptoms per se. In addition, patients investigated in those studies had documented pathological acid reflux while our study population had only "physiological" occurrences of acid reflux and not GORD, as assessed by prior 24 hour pHmetry. The increased vagal activity noted during acid infusion is not unique as we have reported the same with mechanical and electrical oesophageal stimulation. ${ }^{17}$ We believe that the increased vagal activity elicited by oesophageal stimuli, whether electrical, mechanical, or by acid administration, constitutes a pseudoaffective response associated with perception of the oesophageal stimulus, in keeping with the original concepts proposed by Woodsworth and Sherrington. ${ }^{29}$ However, this vagal reflex response occurs with painful as well as non-painful stimuli, provided these are sufficiently intense to be perceived. This suggests that under certain circumstances, non-noxious stimuli can elicit a reflex response akin to the pseudoaffective response elicited by a painful stimulus. This brings into question the specificity of pseudoaffective responses to nociceptive stimuli. $^{30}$ We would instead propose that the awareness of a potentially noxious visceral stimulus, even if its intensity has not yet become painful, is sufficient to elicit a pseudoaffective response. However, only stimuli of a potentially noxious nature (such as acid or distension), that are sufficiently intense enough to be perceived, appear to elicit the cardiac vagoefferent response we describe.

Whether the reflex is due to prior sensitisation of the oesophageal sensory pathways cannot be ascertained in the present study. However, Mehta and colleagues ${ }^{31}$ have shown oesophageal sensitisation to mechanical stimulation following prior perfusion with hydrochloric acid in NCCP patients and a lowered threshold for pain induced by balloon distension after acid perfusion. There may be a similar mechanism involved in acid sensitive patients.

Could there be a causal relationship between the altered sympathovagal activity we observed in acid sensitive patients and the symptoms induced by oesophageal acid perfusion? There is substantial evidence to support the concept of sympathetic modulation of visceral perception although the mechanisms are poorly understood. ${ }^{32}$ Chronic inflammation or even a prior inflammatory mucosal insult may be important factors in conditions such as sympathetically mediated pain and reflex sympathetic dystrophy. ${ }^{33}$ While primarily associated with somatic pain, these entities have also been implicated in visceral pain syndromes. ${ }^{34}$ This possibility deserves further investigations, such as pharmacological manipulations, in NCCP patients. It could be that previous mucosal acid injury elicits alteration in oesophageal sensory neurones or abnormal sensory processing at the level of the spine in susceptible patients. These alterations would in turn produce the altered visceral perception (pain) we observed in acid sensitive NCCP patients. However, these mechanisms fail to adequately explain the decreased vagal activity that is observed in addition to the trend towards increased sympathetic modulation seen in acid sensitive patients prior to acid challenge.

Stress and anxiety are associated with an increased sympathetic activity and decreased vagal activity. ${ }^{35}$ Other functional disorders, such as non-ulcer dyspepsia, have also been associated with decreased vagal activity. ${ }^{35}$ There is substantial evidence to support a vagoafferent modulation of visceral nociception. ${ }^{14}$ This vagoafferent modulation is very likely interacting with efferent vagal activity through central as well as peripheral loci, including the periaqueductal gray, which is essential to the coordinated behavioural and autonomic response to visceral stimulus. ${ }^{36}$ As the periaqueductal gray region has been found to modulate the nociceptive response elicited at the level of spinal afferents, it may represent a key site in the autonomic modulation of visceral perception in patients with functional symptoms.

Power spectral analysis of heart rate variability requires steady state conditions. To meet these standards an assumption is made that during oesophageal acid infusion there is little or no effect on cardiovascular stationarity. Heart rates did not change for either acid sensitive or acid insensitive groups throughout the acid infusion period, nor did they for the control group. Moreover, all groups underwent identical protocols including the same amount of acid infusion over similar time intervals and yet a significant shift in $\mathrm{HF}$ power was seen in one group and not the other. Because the groups began with different baseline power spectral values, the possibility of regression towards the mean for the acid sensitive groups has to be considered. This is unlikely in view of the recovery data that clearly show a reversal of the vagal effect following discontinuation of the infusion.

The present study cannot assess whether the altered resting autonomic balance and the increased vagal response to oesophageal acidification observed in acid sensitive NCCP patients is unique to this group of patients. Whether similar changes are observed in the heterogeneous group of patients reporting more common reflux symptoms such as heartburn remains to be determined. However, patient selection will be paramount to ensure a fairly homogeneous sample in terms of severity of acid reflux and mucosal damage, among others.

In conclusion, we have identified significant differences in cardiac autonomic function between acid sensitive and insensitive NCCP patients. Acid sensitive subjects exhibit a vagally mediated oesophagocardiac reflex alteration in heart rate variability during acid associated symptoms which is not seen in those without symptoms. Increased basal sympathetic activity and/or decreased basal vagal tone are underlying neural abnormalities associated with oesophageal hypersensitivity to acid exposure, possibly the consequence of longstanding psychophysical factors associated with anxiety and stress. 
This work was supported by a grant from the Medical Research Council of Canada to Dr Gervais Tougas. Dr Tougas and Dr Kamath contributed equally to this manuscript.

1 Cannon RO III, Benjamin SB. Chest pain as a consequence of abnormal visceral nociception. Dig Dis Sci 1993;38:1936.

2 Richter JE, Bradley LA, Castell DO. Esophageal chest pain: current controversies in pathogenesis, diagnosis, and therapy. Ann Intern Med 1989;110:66-78.

3 Loomis CW, Yao DY, Bieger D. Characterization of an esophagocardiovascular reflex in the rat. Am F Physiol Regul Integr Comp Physiol 1997;272:R1783-91.

4 Tougas G, Wang L. Pseudoaffective cardioautonomic responses to gastric distension in rats. Am F Physiol Regul Integr Comp Physiol 1999;277:R272-8.

5 Cevese A, Mary DASG, Poltronieri R, et al. Haemodynamic effects of distention of the descending colon in anaesthetized dogs. F Physiol (Lond) 1992;447:409-23.

6 Richter JE, Barish CF, Castell DO. Abnormal esophageal perception in patients with esophageal pain. Gastroenterology 1986;91:845-52.

7 Klinkenberg-Knol EC, Castell DO. Clinical spectrum and diagnosis of gastroesophageal reflux disease. In: Castell DO, ed. The esophagus, 3rd Edn. Philadelphia: Lippincott, DO, ed. The esophagus, 3rd Edn. Philad

8 Watson RGP, Tham TCK, Johnston BT, et al. Double blind cross-over placebo controlled study of omeprazole in the treatment of patients with reflux symptoms and physiological levels of acid reflux-The "sensitive oesophagus". Gut 1997;40:587-90.

9 Rao SSC, Gregersen H, Hayek B, et al. Unexplained chest pain: the hypersensitive, hyperreactive, and poorly compliant esophagus. Ann Intern Med 1996;124:950-8.

10 Galmiche JP, Scarpignato C. Oesophageal sensitivity to acid in patients with non-cardiac chest pain: is the oesophagus hypersensitive? Eur 7 Gastroenterol Hepatol 1995;7:1152-9.

11 Cervero F, Janig W. Visceral nociceptors: a new world order? TINS 1992;15:375-8.

12 Schott GD. Visceral afferents: Their contribution to 'sympathetic dependent' pain. Brain 1994;117:397-413.

13 Green PG, Miao FJ, Strausbaugh H, et al. Endocrine and vagal controls of sympathetically dependent neurogenic vagal controls of sympathetically dependent ne
inflammation. Ann NY Acad Sci 1998;840:282-8.

14 Randich A, Gebhart GF. Vagal afferent modulation of nociception. Brain Res Brain Res Rev 1992;17:77-99.

15 Diop L, Rivière PJM, Pascaud X, et al. Role of vagal afferents in the antinociception produced by morphine and $\mathrm{U}-50,488 \mathrm{H}$ in the colonic pain reflex in rats. Eur $\mathcal{F}$ Pharmacol 1994;257:181-7.

16 Kamath MV, Fallen EL. Power spectral analysis of heart rate variability: a noninvasive signature of cardiac autonomic function. Crit Rev Biomed Eng 1993;21:245-311.

17 Tougas G, Kamath M, Watteel G, et al. Modulation of neurocardiac function by oesophageal stimulation in humans. Clin Sci 1997;92:167-74.

18 Pagani M, Lombardi F, Guzetti F, et al. Power spectral analysis of heart rate and arterial pressure variabilities as a marker of sympathovagal interaction in man and conscious dogs. Circ Res 1986;59:178-93.
19 Malliani A, Pagani M, Furlan R, et al. Individual recognition by heart rate variability of two different autonomic profiles related to posture. Circulation 1997;96:4143-5.

20 Nostrant TT. Provocation testing in noncardiac chest pain Am f Med 1992;92:56-64S.

21 Kamath MV, Upton ARM, Talalla A, et al. Neurocardiac responses to vagoafferent electrostimulation in humans. PACE Pacing Clin Electrophysiol 1992;15:1581-7.

22 Bajwa A, Hollerbach S, Kamath MV, et al. Neurocardiac response to esophageal electric stimulation in humans: Effects of varying stimulation frequencies. Am 7 Physiol Regul Integr Comp Physiol 1997;272:R896-901.

23 Tougas G, Hudoba P, Fitzpatrick D, et al. Cerebral-evoked potential responses following direct vagal and esophageal electrical stimulation in humans. Am f Physiol 1993;264: G486-91.

24 Upton ARM, Tougas G, Talalla A, et al. Neurophysiological effects of left vagal stimulation in man. PACE Pacing Clin Electrophysiol 1991;14:70-6.

25 Ryan SM, Goldberger AC, Mietus J, et al. Gender- and agerelated differences in heart rate dynamics: are women more complex than men! f Am Coll Cardiol 1994;24:1700-7.

26 Harvey A, Kamath MV, McCartney N, et al. Age dependent changes in neurocardiac function in healthy males and females. Can f Cardiol 1998;14:126F.

27 Cunningham KM, Horowitz M, Riddell PS, et al. Relations among autonomic nerve dysfunction, oesophageal motility, and gastric emptying in gastro-oesophageal reflux disease. Gut 1991;32:1436-40.

28 Chakraborty TK, Ogilvie AL, Heading RC, et al. Abnormal cardiovascular reflexes in patients with gastro- oesophageal reflux. Gut 1989;30:46-9.

29 Woodsworth RS, Sherrington CS. A pseudoaffective reflex and its spinal path. F Physiol (Lond) 1904;31:234-43.

30 Malliani A. The autonomic nervous system: A Sherringtonian revision of its integrated properties in the control of circulation. F Auton Nerv Syst 1997;64:158-61.

31 Mehta AJ, De Caestecker JS, Camm AJ, et al. Sensitization to painful distention and abnormal sensory perception in the esophagus. Gastroenterology 1995;108:311-19.

32 Blumberg H, Haupt P, Janig W, et al. Encoding of visceral noxious stimuli in the discharge patterns of visceral afferent fibres from the colon. Pflugers Arch 1983;398:33-40.

33 Blumberg $\mathrm{H}$, Jaenig W. Clinical manifestations of reflex sympathetic dystrophy and sympathetically mediated pain. In: Wall PD, Melzack R, eds, Textbook of pain, 3rd Edn. Edinburgh: Churchill Livingstone, 1994:685-98.

34 Janig W. Neurobiology of visceral afferent neurons: neuroanatomy, functions, organ regulations and sensations. Biol anatomy, functions, org
Psychol 1996;42:29-51.

35 Haug TT, Wilhelmsen I, Berstad A, et al. Life events and stress in patients with functional dyspepsia compared with patients with duodenal ulcer and healthy controls. Scand $\mathcal{F}$ Gastroenterol 1995;30:524-30.

36 Bandler R, Carrive P, Zhang SP. Integration of somatic and autonomic reactions within the midbrain periaqueductal grey: viscerotopic, somatotopic and functional organization. Prog Brain Res 1991;87:269-305.

\section{9th United European Gastroenterology Week}

The UEGW abstract book (Gut 2001;49(suppl III)) has again been produced as a CD-ROM and can be found attached to the inside back cover of this issue. 